doi: $10.17951 /$ a.2019.73.2.1-3

A N N A LES

UNIVERSITATIS MARIAE CURIE-SKŁODOWSKA

L UBLIN - POLONIA

VOL. LXXIII, NO. 2, 2019

SECTIO A

$1-3$

\title{
Scientific life of Professor Yuri Kozitsky
}

This special issue of the Annales Universitatis Mariae Curie-Sktodowska, Sectio A - Mathematica is dedicated to Professor Yuri Kozitsky on the occasion of his 70th birthday. Yuri Kozitsky is a renowned scholar in the field of dynamical systems and mathematical background of statistical physics. His seminal works on fundamental problems in these fields as well as on applications of dynamical system ideas in biology, ecology, medicine are well known and recognized by the community.

Yuri Kozitsky was born on April 21, 1949 in Krukiv (now Kremenchuk), Ukraine. He graduated from the secondary school in the city of Kremenchuk, and in 1967 became a student of the physical department of the Ivan Franko State University in Lviv. Admired by two sciences: physics and mathematics, he chose his specialization in the field of theoretical solid state physics. His diploma thesis, supervised by Prof. Ihor Stasyuk was devoted to studies of dynamical properties of ferroelectric crystals with hydrogen bonds. After doctoral studies in the Lviv Division of Kyiv's Institute for Theoretical Physics of the Academy of Sciences of Ukraine under supervision of Prof. Ihor Yukhnovskii, in 1981 he got the $\mathrm{PhD}$ degree in theoretical and mathematical physics, defending the thesis entitled Applications of the method of collective variables to the study of scalar models of phase transitions in which hierarchical models of many-particle systems were used to explain critical behaviour of condensed matter. The thesis was based in part on novel ideas of renormalization that arrived to statistical physics from quantum field theory. Already in this thesis one can trace particular features of Yuri Kozitsky's style, that joins mathematical rigor with modeling in order to explain newly observed or well-known but still unclear natural phenomena. In parallel, he lectured mathematics in the Lviv Academy of Commerce, first as an assistant (1974-1982), senior lecturer (1982-1984) and then as 
a professor and chair for higher mathematics (1984-1996). In 1992, he obtained the Doctor of science (habilitation) degree in mathematical analysis, defending the dissertation entitled Laguerre entire functions in problems of mathematical physics in the Institute of Mathematics of the National Academy of Sciences of Ukraine (Kyiv). Next, in 1994, he received Professorship in Mathematics from the Ministry of Education of Ukraine.

Since 1996 up to the present time Yuri Kozitsky has held the position of full professor in the Institute of Mathematics of Maria Curie-Skłodowska University in Lublin, Poland. There, he leads a school of applications of mathematics, having prepared a number of talented researches in this field. In Lublin he chairs a research seminar and has supervised seven doctoral students. He also maintains intensive scientific contacts with his colleagues, physicists and mathematicians from Germany, Israel, Great Britain, Ukraine, Romania and other countries. He inspired and organized a series of conferences Infinite particle systems in Kazimierz Dolny (Poland), led prestigious international collaboration projects, held invited research and lecturing positions in many countries all over the world. Beneath them Forschungszentrum BiBoS, Bielefeld and Ruhr University Bochum (Germany), University of Rome La Sapienza (Italy), ORT Braude College, Karmiel (Israel), Institute for Condensed Matter Physics, Lviv (Ukraine), University of Bucharest (Romania), Simeon Stoilow Institute of Mathematics of Romanian Academy, etc. He is a member of Editorial boards of the journals Condensed Matter Physics, International Journal: Mathematical Manuscripts, International Review of Physics.

Physics and mathematics have fascinated Yuri Kozitsky from the early beginning of his studies, and he remains faithful to them throughout his long and fruitful life in science. His scientific interests cover stochastic dynamics of infinite particle systems, mathematical problems of statistical physics, Laguerre entire functions and their applications, nonlinear holomorphic mappings in topological spaces, integral and differential operators and functional differential equations, quantum informatics, applications of dynamical systems in biology, ecology, medicine. This list is not completed and is still increasing! His studies of mathematical foundations of theoretical approaches in condensed matter physics provided reliable background and verification for a number of results obtained phenomenologically. In particular, he provided rigorous verification of the self-similarity symmetry in the theory of second order phase transitions. The implementation of this concept in the collective variables approach opened new possibilities for constructing the hierarchical models starting from the lattice models of ferromagnets. He contributed also to quantum statistical mechanics a theory that plays the major role in many fields such as thermodynamics, plasma physics, solid-state physics, and the study of stellar structure. While the theory of quantum harmonic oscillators is relatively simple, the 
case of anharmonic oscillators, a mathematical model of a localized quantum particle, is more complex and challenging and was considered by Yuri Kozitsky and his colleagues. These results are summarized in the book he coauthored with Sergio Albeverio, Yuri Kondratiev and Michael Roeckner (The Statistical Mechanics of Quantum Lattice Systems: A Path Integral Approach, EMS Tracts in Mathematics 8, European Mathematical Society (EMS), 2009).

As we have already mentioned, the spectrum of Yuri Kozitsky's scientific, in particular, mathematical interests is very wide. At the beginning of his mathematical career, he studied and investigated some subjects related to the theory of holomorphic mappings in finite dimensional spaces. Later during his sabbatical in Israel, he was involved in the project on numerical range of holomorphic mappings and nonlinear semigroup theory in Banach and Hilbert spaces, where his contribution to linear and nonlinear operator equations was very essential. Together with Israeli and Italian mathematicians, he published papers, which give new perspectives for future developments in the infinite dimensional holomorphy and nonlinear operator theory. At that time, he collaborated in ORT Braude College in Israel with Mark Elin, Fiana Jacobson, Marina Levenstein, and David Shoikhet as well as with Filippo Bracci from University Rome 2, who visited Israel at that period.

On behalf of the editorial board of the Annales Universitatis Mariae Curie-Skłodowska, numerous colleagues and friends it is our great honour and pleasure to congratulate Professor Yuri Kozitsky on his jubilee and to wish him further long and successful life in science.

Yurij Holovatch (Lviv),

Stanisław Prus (Lublin),

David Shoikhet (Karmiel/Holon) 